\title{
Modelling of natural growth with memory effect in economics: An application of adomian decomposition and variational iteration methods
}

\author{
Muhamad Deni Johansyaha ${ }^{a}$, Asep K. Supriatna ${ }^{a}$, Endang Rusyamana and Jumadil Saputra ${ }^{b^{*}}$
}

${ }^{a}$ Faculty of Mathematics and Natural Sciences, Universitas Padjadjaran, Jatinangor, Sumedang, Jawa Barat 45363 Indonesia ${ }^{b}$ Faculty of Business, Economics and Social Development, Universiti Malaysia Terengganu, 21030 Kuala Nerus, Terengganu, Malaysia

\begin{tabular}{l}
\hline C H R O N I C L E \\
\hline Article history: \\
Received May 5, 2021 \\
Received in revised format: \\
June 1, 2021 \\
Accepted August 182021 \\
Available online \\
August 19, 2021 \\
\hline Keywords: \\
Natural growth models \\
Memory effect in economics \\
Adomian Decomposition and \\
Variational Iteration methods \\
Numerical simulation analysis \\
Decision science
\end{tabular}

\begin{abstract}
A B S T R A C T
The power-law memory effect is taken into consideration in a generalisation of the economic model of natural growth. The memory effect refers to a process's reliance on its current state and its history of previous changes. However, the study that focuses on natural growth in economics considering the memory effect with fractional order-linear differential equation model is still limited. The current investigation seeks to solve the natural growth with memory effect in the economics model and decide the best model using fractional differential equation (FDE), namely Adomian Decomposition and Variational Iteration Methods. Also, this study assumes the level of consumer loss memory during a certain time interval denoted by a parameter $(\alpha)$. This study showed the model of loss memory effect with $0<\alpha \leq 1$ given a slowdown in output growth compared to a model without memory effect. Besides that, this study also found that output $Y(t)$ is growing faster with the Variational Iteration method compared to the Adomian decomposition method. Also, using graphical simulation, this study found the output $Y(t)$ is closer to the exact solution with $\alpha=0.4$ and $\alpha=0.9$. In conclusion, this study successfully solved natural growth with memory effect in economics and decided the best model between FDE, namely Adomian decomposition and Variational iterative methods using numerical analysis.
\end{abstract}

(C) 2022 by the authors; licensee Growing Science, Canada.

\section{Introduction}

In general, growth is calculated in real terms, namely inflation-adjusted terms (Anidiobu, Okolie \& Oleka, 2018). It aims to eliminate the distorting effect of inflation on the prices of goods produced. Since economic growth is measured using the annual percentage changes in the gross domestic product (GDP), it is the advantages and drawbacks of the measurement tool (Sukono, Albra, Zulham, Saputra, Subartini, Thalia, 2019; Sukono, Subartin, Ambarwati, Saputra, Hidayat, 2019). Among two economists' points of view to the growth model, Solow stated that the actual growth rate converges on the natural rate, whatever the saving-to-income ratio. Besides that, Harrod-Domar stated that the warranted growth rate is higher than the natural rate, the economy will continue to grow until full employment is achieved. There will be a recession if real output growth falls below the required rate and will tend to ever-increasing unemployment if the authorised growth rate falls below the natural rate.

Nowadays, the natural growth model is currently applied to natural science and economics. The model explained by an equation whose output growth rate is proportional to income. A more reliable model is a natural growth that relies more on profits compared to income. Numerous previous studies have discussed the natural growth models in economics without lags effect (e.g., Wainwright, 2005; Volterra, 2005) and loss memory effects (e.g., Tarasov and Tarasova, 2021; Tarasova and Tarasov, 2016; Tarasova and Tarasov, 2016). In this study, we suppose that $Y(t)$ is a function that describes the value of output at time $t$. Further, $I(t)$ is a function which states a net investment used for the development of production. In the

* Corresponding author.

E-mail address: jumadil.saputra@umt.edu.my (J. Saputra)

(C) 2022 by the authors; licensee Growing Science, Canada. doi: $10.5267 /$ j.dsl.2021.8.003 
natural growth model in economics, it assumed that marginal income $\left(\frac{d P Y(t)}{d t}\right)$ and output, $\left(\frac{d Y(t)}{d t}\right)$ are proportional to the net investment value. Thus, the acceleration equation can be written as follow:

$$
\frac{d Y(t)}{d t}=\frac{1}{v} I(t)
$$

whereas $v$ is the investment ratio in the form of a positive constant, which describes the acceleration power. Also, $\frac{1}{v}$ is the marginal productivity of capital (acceleration), and $\frac{d Y(t)}{d t}$ is the first derivative order at time $t$ of $Y(t)$. It is assumed that net investment value is a fixed part of the profit that is proportional to the difference between income $P Y(t)$ and cost $C(t)$. Thus, it can be obtained from the following equation:

$$
I(t)=m(P Y(t)-C(t))
$$

where $m$ is the rate of net investment $(0<m<1)$, namely the distribution of profits used for net investment. It is assumed that $C(t)$ is a linear cost function with output $Y(t)$, thus obtained:

$$
C(t)=a Y(t)+b
$$

where $a$ is the marginal cost and $b$ is the independent cost, which is part of the cost that does not rely on the value of output. Eq. (2) and Eq. (3) are substituted to Eq. (1) then the following are obtained:

$\frac{d Y(t)}{d t}=\frac{1}{v}(m(P Y(t)-C(t)))=\frac{m}{v}(P Y(t)-(a Y(t)+b))=\frac{m P Y(t)}{v}-\frac{m(a Y(t)+b)}{v}=\frac{m P Y(t)}{v}-\frac{m a Y(t)}{v}-\frac{m b}{v}$

so that

$$
\begin{aligned}
\frac{d Y(t)}{d t}-\frac{m P Y(t)}{v}+\frac{m a Y(t)}{v} & =-\frac{m b}{v} \\
\frac{d Y(t)}{d t}-\frac{m}{v}(P-a) Y(t) & =-\frac{m b}{v} .
\end{aligned}
$$

Let's,

$\lambda_{1}=\frac{m}{v}(P-a), \lambda_{2}=-\frac{m b}{v}$

Thus, Eq. (4) is the first-order linear differential equations and can be simplified as follow:

$$
\frac{d Y(t)}{d t}-\lambda_{1} Y(t)=\lambda_{2}
$$

Eq. (5) is rewritten by:

$Y^{\prime}(t)+Q(t) Y(t)=r(t)$

Thus, the exact solution can be obtained by using the below equations:

$Y(t)=e^{-h}\left(\int e^{h} r(t) d t+k\right)$

with $\int Q(t) d t=\int-\lambda_{1} d t=-\lambda_{1} t$, so

$Y(t)=e^{\lambda_{1} t}\left(\int e^{-\lambda_{1} t} \lambda_{2} d t+k\right)=e^{\lambda_{1} t}\left(-\frac{\lambda_{2}}{\lambda_{1}} e^{-\lambda_{1} t}+k\right)=-\frac{\lambda_{2}}{\lambda_{1}}+e^{\lambda_{1} t} k$

Thus, the general solution of Eq. (4) is as follows:

$$
Y(t)=\frac{b}{P-a}+k e^{\frac{m}{v}(P-a) t}
$$


So, when we use the initial value $Y(0)$ into Eq. (6) for $t=0$, the value of $k$ is obtained as

$k=Y(0)-\frac{b}{P-a}$

By substituting the value of $\mathrm{k}$ into Eq. (6), the following equation is obtained:

$$
Y(t)=\frac{b}{P-a}\left(1-e^{\frac{m}{v}(P-a) t}\right)+Y(0) e^{\frac{m}{v}(P-a) t}
$$

Eq. (7) is the general solution Eq. (4) describes the output dynamics of natural growth models without memory effects. Referring to (Tarasova and Tarasov, 2016), the natural growth model given by Eq. (4) is expected to produce an acceleration Eq. (1) which is related to the value of the net investment and marginal output. Eq. (1) and Eq. (4) are the first-order derivative equation concerning time. The first-order derivative is set by the character of differentiable function toward time around the very small neighbourhood of time point. Thus, the natural growth model of Eq. (4) ignores the memory effect and time lag. In this research, Eq. (4) is developed into a fractional differential equation with order $\alpha$ that involve the memory effect. Memory effects are considered properties that describe independent variables that originate from the current process and constitute changes in variables in the past at certain periods of time, $t$ (Tarasova \&Tarasov, 2016). To explain the economic process, derivatives and fractional differential equations are used in $\alpha$ order.

\section{The Memory Effect with Fractional Derivatives and Integrals}

The idea of dynamic memory to represent the economic processes and concepts have been applied in physics (Tarasov, 2011). Dynamic memory is a common characteristic of processes that describe the current state of the process depending on the state of processes in the past (Tarasov \& Tarasova, 2021; Tarasova \& Tarasov, 2016). Furthermore, the concept of memory effects is described for economic growth. The economic process is a process in which the parameters and economic factors (endogenous and exogenous variables) at a given time rely not on only the current values but also relies on the value at the previous time (Tarasova \& Tarasov, 2016; Valentina \& Vasily, 2016). To include the effect of memory in the natural economic growth model, it is assumed that the output value $Y(t)$ at time $t$ relies not on only the net investment $I(t)$ at the same time point, but also relies on the changes $I(\tau)$ at a finite time interval $[0, t]$. The economic actors can remember changes in former investments $I(t)$ and its changes affect of output value $Y(t)$. To illustrate the memory effect (refer to Eq. (8)) as below:

$$
Y(t)=\int_{0}^{t} M(t-\tau) I(\tau) d \tau
$$

with $M(t)$ is a memory function associated with the memory of the net investment norms (Tarasova and Tarasov, 2016). If the function $M(t)$ described by Dirac delta function $(M(t)=M \delta(t))$, thus Eq. (8) become a standard multiply equation $Y(t)=M I(t)$. Whenever $M(t)$ have a form $M(t)=M \delta(t-\tau)$, thus Eq. (8) be a multiplier formula with fixed time lag $Y(t)=M I(t-\tau)$, (Takayama and Akira, 1985).

If the normalise condition $\int_{0}^{t} M(t-\tau) d \tau=1$ holds for $M(t)$. Thus Eq. (8) can be considered the formula with a time lag, which is continuously distributed (so called the weight). Assuming a fractional derivative rule with the order $\alpha$ which involves the effect of memory loss, the memory function can be used as follow:

$$
M(t-\tau)=\frac{1}{\Gamma(\alpha)} \frac{M}{(t-\tau)^{1-\alpha}}
$$

With $\alpha>0, t>\tau$, and $\Gamma(\alpha)$ is the Gamma function. To get the correct dimensions of the form of economic quantity, dimensionless time variable $t$ is used, and Eq. (8) is stated as follow:

$$
Y(t)=\int_{0}^{t} \frac{1}{\Gamma(\alpha)} \frac{M}{(t-\tau)^{1-\alpha}} I(\tau) d \tau=\frac{M}{\Gamma(\alpha)} \int_{0}^{t} \frac{1}{(t-\tau)^{1-\alpha}} I(\tau) d \tau=M\left(J_{0+}^{\alpha} I\right)(t),
$$

where $J_{0+}^{\alpha}$ is the right-hand side of Riemann-Liouville fractional integral with order $\alpha>0$ with variable $\mathrm{t}$ which is expressed by the equation

$$
\left(J_{0+}^{\alpha} I\right)(t)=\frac{1}{\Gamma(\alpha)} \int_{0}^{t} \frac{I(\tau)}{(t-\tau)^{1-\alpha}} d \tau
$$


with $0<\tau<t$, and function $I(\tau)$ at intervals $(0, t)$ and satisfied $\int_{0}^{t}|I(\tau)| d \tau<\infty$. Eq. (11) illustrates the multipliers of fractional order (Tarasova \& Tarasov, 2016), and $M$ is the multiplier coefficient of this equation. To show the net investment function $I(t)$ in output function $Y(t)$, the Eq. (11) with the Caputo fractional derivative with order $\alpha>0$ is used (Podlubny, 1999; Mathai \& Haubold, 2017).

$$
\left(D_{0+}^{\alpha} Y\right)(t)=\frac{1}{\Gamma(n-\alpha)} \int_{0}^{t} \frac{Y^{n}(\tau)}{(t-\tau)^{\alpha-n+1}} d \tau
$$

where $Y^{n}(\tau)$ is a derivative with integer-order $n:=[\alpha]+1$ of function $Y(\tau)$ respect to $\tau$ such that $0<\tau<t$. Function $Y(\tau)$ must have derivatives from the order of integers until the- $(n-1)$, which is a continuous function at intervals $[0, t]$. Derivation of the Eq. (12) of Eq (10) can be represented as:

$$
\left(D_{0+}^{\alpha} J_{0+}^{\alpha} f\right)(t)=f(t)
$$

Eq. (13) applies for every $\alpha>0$, where $J_{0+}^{\alpha}$ is the right side of Riemann-Liouville fractional integral Eq. (10) and $D_{0+}^{\alpha}$ is the right side of the Caputo fractional derivative (12), using the equation of identity Eq. (13), then Eq. (12) can be written:

$$
\left(D_{0+}^{\alpha} Y\right)(t)=M I(t)=\frac{1}{v} I(t)
$$

where $v=\frac{1}{M}$.

\section{Economic Growth Equations with Memory Effects and its Solution}

Considering the effects of memory loss on natural growth models, Eq. (14) is used, which illustrates the connection between net investment and the value of marginal output with order $\alpha>0$ (Mathai \& Haubold, 2017; Tarasov \& Tarasova, 2016; Tarasov \& Tarasova, 2016). Substitutes Eq. (2) and Eq. (3) to Eq. (14). Thus, we obtained that:

$$
\begin{aligned}
\left(D_{0+}^{\alpha} Y\right)(t)=\frac{1}{v}( & m(P Y(t)-C(t)))=\frac{m}{v}(P Y(t)-(a Y(t)+b))=\frac{m P Y(t)}{v}-\frac{m(a Y(t)+b)}{v} \\
= & \frac{m P Y(t)}{v}-\frac{m a Y(t)}{v}-\frac{m b}{v}
\end{aligned}
$$

So that:

$$
\begin{aligned}
\left(D_{0+}^{\alpha} Y\right)(t)-\frac{m P Y(t)}{v}+\frac{m a Y(t)}{v} & =-\frac{m b}{v} \\
\left(D_{0+}^{\alpha} Y\right)(t)-\frac{m}{v}(P-a) Y(t) & =-\frac{m b}{v} .
\end{aligned}
$$

Eq. (15) is a fractional order-linear differential equation $\alpha>0$. The natural economic growth model is based on Eq. (15), which involves memory loss by order $\alpha>0$. The exact solution of Eq. (15) with $\alpha=1$ is the Eq. (7), which describes natural growth models without memory effects. Furthermore, the purpose is to obtain Eq. (15), a natural growth model with the memory loss effect. Eq. (15) is a linear order of fractional differential equations with order $\alpha$. Then, it can be written as below:

$$
\left(D_{0+}^{\alpha} Y\right)(t)-\lambda_{1} Y(t)=\lambda_{2}
$$

where $\lambda_{1}=\frac{m}{v}(P-a), \lambda_{2}=-\frac{m b}{v}$.

\section{The Solution of the Natural Growth Model with Memory Effect}

In this paper, methods for finding solutions to fractional differential equations with order $0<\alpha \leq 1$ with $t>0$ using two methods, namely Adomian Decomposition Method (ADM) and Variational Iteration Method (VIM) as given follows. The basic concepts and theories regarding ADM and VIM (refer to Adomian, 1988; Podlubny, 1998; He, 1999; Gbadamosi, Adebimpe, Akinola, \& Olopade, 2012; Merdan, 2012; Saad, Al-Shomrani, Mohamed, \& Yang, 2016).

\subsection{Adomian Decomposition Method (ADM)}

Eq. (16) can be written as follow:

$$
\left(D_{0+}^{\alpha} Y\right)(t)=\lambda_{1} Y(t)+\lambda_{2}
$$


with $D_{0+}^{\alpha}=\frac{d^{\alpha}}{d t^{\alpha}}$ is the fractional derivative operator with order $\alpha$, where $\left(D_{0+}^{\alpha} Y\right)(t)=\frac{1}{\Gamma(n-\alpha)} \int_{0}^{t} \frac{Y^{n}(\tau)}{(t-\tau)^{\alpha-n+1}} d \tau$. While the inverse of $D_{0+}^{\alpha}$ is $D_{0+}^{-\alpha}=J^{\alpha}$. Eq. (17) multiply by $D_{0+}^{-\alpha}$ obtained:

$$
\begin{aligned}
D_{0+}^{-\alpha}\left(D_{0+}^{\alpha} Y\right)(t) & =D_{0+}^{-\alpha}\left(\lambda_{1} Y(t)+\lambda_{2}\right) \\
Y(t)-Y(0) & =J^{\alpha} \lambda_{1} Y(t)+J^{\alpha} \lambda_{2} \\
Y(t) & =Y(0)+J^{\alpha} \lambda_{1} Y(t)+J^{\alpha} \lambda_{2} .
\end{aligned}
$$

Adomian Decomposition Method assumes that function $Y(t)$ can be decomposed into infinite series as follows $Y(t)=$ $\sum_{n=0}^{\infty} Y_{n}(t)$. Thus, Eq. (18) can be written as:

$$
\sum_{n=0}^{\infty} Y_{n}(t)=Y(0)+\lambda_{1} J^{\alpha} \sum_{n=0}^{\infty} Y_{n}(t)+J^{\alpha} \lambda_{2}
$$

Based on Eq. (19), the solution of a fractional differential Eq. (16) can be formed into the following recursive relation, as seen as follow:

$Y_{0}=Y(0)+J^{\alpha} \lambda_{2}$

$Y_{n+1}=\lambda_{1} J^{\alpha} Y_{n}, n=1,2,3, \ldots$

With,

$Y_{0}=Y(0)+J^{\alpha} \lambda_{2}=Y(0)+\frac{t^{\alpha}}{\Gamma(\alpha+1)} \lambda_{2}$

$Y_{1}=\lambda_{1} J^{\alpha} Y_{0}=\lambda_{1} J^{\alpha}\left(Y(0)+\frac{t^{\alpha}}{\Gamma(\alpha+1)} \lambda_{2}\right)=\frac{t^{\alpha}}{\Gamma(\alpha+1)} \lambda_{1} Y(0)+\frac{t^{2 \alpha}}{\Gamma(2 \alpha+1)} \lambda_{1} \lambda_{2}$

$Y_{2}=\lambda_{2} J^{\alpha} Y_{1}=\lambda_{1} J^{\alpha}\left(\frac{t^{\alpha}}{\Gamma(\alpha+1)} \lambda_{1} Y(0)+\frac{t^{2 \alpha}}{\Gamma(2 \alpha+1)} \lambda_{1} \lambda_{2}\right)=\frac{t^{2 \alpha}}{\Gamma(2 \alpha+1)} \lambda_{1}^{2} Y(0)+\frac{t^{3 \alpha}}{\Gamma(3 \alpha+1)} \lambda_{1}^{2} \lambda_{2}$

$Y_{3}=\lambda_{2} J^{\alpha} Y_{2}=\lambda_{1} J^{\alpha}\left(\frac{t^{2 \alpha}}{\Gamma(2 \alpha+1)} \lambda_{1}^{2} Y(0)+\frac{t^{3 \alpha}}{\Gamma(3 \alpha+1)} \lambda_{1}^{2} \lambda_{2}\right)=\frac{t^{3 \alpha}}{\Gamma(3 \alpha+1)} \lambda_{1}^{3} Y(0)+\frac{t^{4 \alpha}}{\Gamma(4 \alpha+1)} \lambda_{1}^{3} \lambda_{2}$

Thus,

$Y(t)=\sum_{n=0}^{\infty} Y_{n}(t)=Y_{0}+Y_{1}+Y_{2}+\cdots=Y(0) \sum_{n=0}^{\infty} \frac{\lambda_{1}^{n} t^{n \alpha}}{\Gamma(n \alpha+1)}+\lambda_{2} \sum_{n=0}^{\infty} \frac{\lambda_{1}^{n} t^{(n+1) \alpha}}{\Gamma((n+1) \alpha+1)}$,

So, we obtained that:

$$
Y(t)=Y(0) \sum_{n=0}^{\infty}\left(\frac{m}{v}(P-a)\right)^{n} \frac{t^{n \alpha}}{\Gamma(n \alpha+1)}-\frac{m b}{v} \sum_{n=0}^{\infty}\left(\frac{m}{v}(P-a)\right)^{n} \frac{t^{(n+1) \alpha}}{\Gamma((n+1) \alpha+1)} .
$$

Eq. (20) is a natural growth model solution that involves memory loss effect using Adomian Decomposition Method. Equation (20) illustrates the growth and decrease in the output value $\mathrm{Y}(\mathrm{t})$ of the natural growth model by involving the effect of memory loss at $0<\alpha \leq 1$ (See Example 1).

\subsection{Variational Iteration Method}

Eq. (16) can be written as:

$$
\left(D_{0+}^{\alpha} Y\right)(t)-\lambda_{1} Y(t)-\lambda_{2}=0
$$

with $D_{0+}^{\alpha}=\frac{d^{\alpha}}{d t^{\alpha}}$ is the fractional derivative operator with order $0<\alpha \leq 1$, where $\lambda_{1}$ and $\lambda_{2}$ be the constants. According to Variational Iterative Method, Eq. (21) can be written as:

$Y_{n+1}=Y_{n}+J^{\alpha} \lambda(\xi)\left[D_{0+}^{\alpha} Y_{n}(t)-\lambda_{1} Y_{n}(t)-\lambda_{2}\right]$ 
with $\lambda(\xi)=-1$, thus:

$Y_{n+1}=Y_{n}-J^{\alpha}\left[D_{0+}^{\alpha} Y_{n}(t)-\lambda_{1} Y_{n}(t)-\lambda_{2}\right]$

The initial condition chosen is $Y_{0}(t)=Y_{0}$. Thus, the next iteration is:

$$
\begin{aligned}
Y_{1} & =Y_{0}-J^{\alpha}\left[D_{0+}^{\alpha} Y_{0}(t)-\lambda_{1} Y_{0}(t)-\lambda_{2}\right]=Y_{0}-J^{\alpha} D_{0+}^{\alpha} Y_{0}(t)+J^{\alpha} \lambda_{1} Y_{0}(t)+J^{\alpha} \lambda_{2}=Y(0)+\frac{t^{\alpha}}{\Gamma(\alpha+1)}\left(\lambda_{1} Y(0)+\lambda_{2}\right) \\
Y_{2} & =Y_{1}-J^{\alpha}\left[D_{0+}^{\alpha} Y_{1}(t)-\lambda_{1} Y_{1}(t)-\lambda_{2}\right]=Y(0)+\frac{t^{\alpha}}{\Gamma(\alpha+1)}\left(\lambda_{1} Y(0)+\lambda_{2}\right)+\frac{t^{2 \alpha}}{\Gamma(2 \alpha+1)}\left(\lambda_{1}^{2} Y(0)+\lambda_{1} \lambda_{2}\right) \\
Y_{3} & =Y_{2}-J^{\alpha}\left[D_{0+}^{\alpha} Y_{2}(t)-\lambda_{1} Y_{2}(t)-\lambda_{2}\right] \\
& =Y(0)+\frac{t^{\alpha}}{\Gamma(\alpha+1)}\left(\lambda_{1} Y(0)+\lambda_{2}\right)+\frac{t^{2 \alpha}}{\Gamma(2 \alpha+1)}\left(\lambda_{1}^{2} Y(0)+\lambda_{1} \lambda_{2}\right)+\frac{t^{3 \alpha}}{\Gamma(3 \alpha+1)}\left(\lambda_{1}^{3} Y(0)+\lambda_{1}^{2} \lambda_{2}\right)
\end{aligned}
$$

Thus, an approximation solution to the Eq. (21) is obtained:

$$
\begin{aligned}
Y(t) \cong Y_{3}(t)= & Y(0)+\frac{t^{\alpha}}{\Gamma(\alpha+1)}\left(\lambda_{1} Y(0)+\lambda_{2}\right)+\frac{t^{2 \alpha}}{\Gamma(2 \alpha+1)}\left(\lambda_{1}^{2} Y(0)+\lambda_{1} \lambda_{2}\right) \\
& +\frac{t^{3 \alpha}}{\Gamma(3 \alpha+1)}\left(\lambda_{1}^{3} Y(0)+\lambda_{1}^{2} \lambda_{2}\right) .
\end{aligned}
$$

Eq. (22) is a solution to the natural growth model that involves memory loss effect using the Variational Iterative Method. Eq. (22) illustrates the growth of the output value $Y(t)$ of the natural growth model involving the loss memory effect at $0<\alpha \leq 1$ (see Example 2).

\section{Numerical Simulation of Natural Growth Models with Memory Effects in Economics}

In this section, a numerical simulation of the natural growth model with memory effect in economics for $\alpha$ equal to 0.9 and 0.4 is presented. Then, we would be comparing and discussing the exact solutions with $(\alpha=1)$.

Example 1. The output value $Y(t)$ at $Y(0)=15, b=3,(P-a)=0.3, m=25$, and $v=40$ for Eq. (21) and Eq. (22) with the order of memory loss $\alpha=0.9$ are presented respectively (see Figure 1), i.e. graph $Y_{A D M}$ and $Y_{V I M}$. It shows the memory loss effect with order $\alpha=0.9$ in both methods affected the slowdown of growth output value $Y(t)$ when compared to the model without memory effects (order $\alpha=1$ ). However, growth in output values $Y(t)$ using ADM is slower than VIM (for $t=10$ the value of $Y_{E X A C T}=42.60409560, Y_{A D M}=35.40457519$, dan $Y_{V I M}=35.85777553$ ).

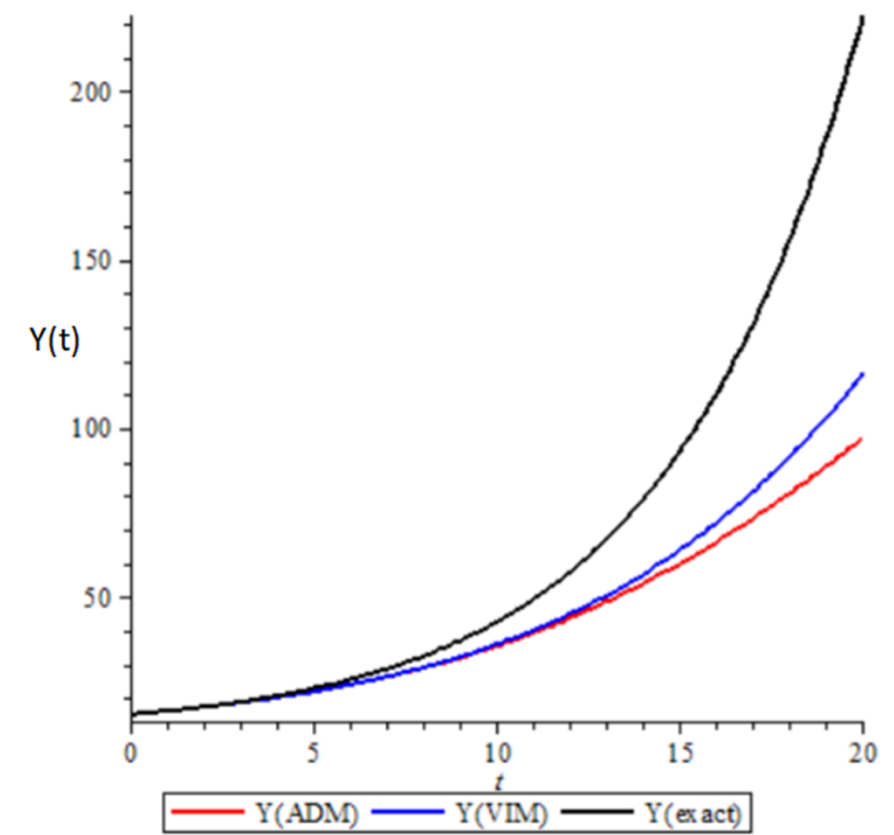

Fig. 1. Graphical output $Y(t)$ with $\alpha=0.9$ for $Y(0)=15, b=3,(P-a)=0.3, m=25, v=40$ 
Example 2. The output value $Y(t)$ at $Y(0)=15, b=3,(P-a)=0.3, m=25$, and $v=40$ for Eq. (20) and Eq. (22) with the order of memory loss $\alpha=0.4$ are presented respectively in Figure 2 graph $Y_{A D M}$ and $Y_{V I M}$. The memory loss effect with order $\alpha=0.4$ in both methods affected the slowdown of growth output value $Y(t)$ when compared to the model without memory effects ( order $\alpha=1$ ).

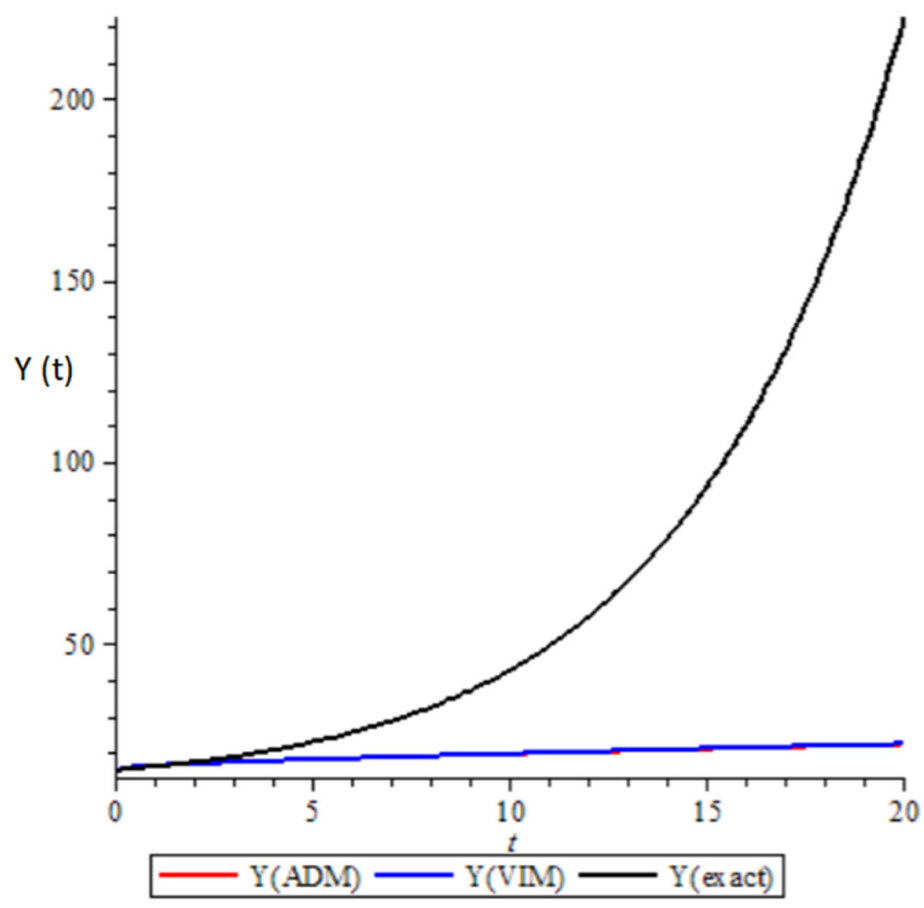

Fig. 2. Graphical output $Y(t)$ with $\alpha=0.4$ for $Y(0)=15, b=3,(P-a)=0.3, m=25, v=40$

Table 1

The numerical simulation using ADM and VIM of output value $\mathrm{Y}(\mathrm{t})$ with $\alpha=0.9$ and $0.4(0 \leq \mathrm{t} \leq 10)$ and its exact solution $\underline{(\alpha=1)}$

\begin{tabular}{cccccc}
\hline \multirow{2}{*}{$t$} & \multicolumn{2}{c}{$\alpha=0.4$} & \multicolumn{2}{c}{$\alpha=0.9$} & \multirow{2}{*}{$Y_{E X A C T}(t)$} \\
\cline { 2 - 4 } & $Y_{A D M}(t)$ & $Y_{V I M}(t)$ & $Y_{A D M}(t)$ & $Y_{V I M}(t)$ & 15.0000 \\
1 & 15.0000 & 15.0000 & 15.0000 & 15.0000 & 16.0311 \\
2 & 16.0880 & 16.0880 & 16.2800 & 16.2801 & 17.2749 \\
3 & 17.2414 & 17.2415 & 16.8061 & 16.8069 & 18.7752 \\
4 & 18.5575 & 18.5582 & 17.2342 & 17.2362 & 20.5850 \\
5 & 20.0754 & 20.0786 & 17.6146 & 17.6186 & 22.7679 \\
6 & 21.8304 & 21.8412 & 17.9660 & 17.9729 & 25.4010 \\
7 & 23.8580 & 23.8867 & 18.2978 & 18.3086 & 28.5772 \\
8 & 26.1938 & 26.2598 & 18.6155 & 18.6310 & 32.4084 \\
9 & 28.8736 & 29.0094 & 18.9223 & 18.9438 & 37.0297 \\
10 & 31.9325 & 32.1891 & 19.2208 & 19.2492 & 42.6040 \\
\hline
\end{tabular}

Table 1 shows the numerical simulation using Adomian Decomposition Method and Variational Iterative Method of output value $Y(t)$ with $\alpha=0.9$ and $0.4(0 \leq t \leq 10)$ and its exact solution $(\alpha=1)$. The numerical results show that the influence of memory with the order of loss $0<\alpha \leq 1$ causing a slowdown in output growth compared to the model without involving memory effects. However, increase in output values $Y(t)$ using ADM is slower than VIM (see for $t=10$ the value of $Y_{E X A C T}=42.60409560, Y_{A D M}=19.51243483$, and $\left.Y_{V I M}=19.54904617\right)$.

\section{Conclusion}

In conclusion, this study successfully solved natural growth with memory effect in economics and decided the best model between FDE, namely Adomian decomposition and Variational iterative methods using numerical analysis. This study found the model of loss memory effect with $0<\alpha \leq 1$ given a slowdown in output growth compared to a model without memory effect. Besides that, the output $\mathrm{Y}(\mathrm{t})$ is growing faster with the Variational Iteration method than the Adomian 
decomposition method. Also, using graphical simulation, this study found the output $\mathrm{Y}(\mathrm{t})$ is closer to the exact solution with $\alpha=0.4$ and $\alpha=0.9$. In addition, we found a numerical solution to the natural growth model with memory effects. Thus, we concluded that the Variational Iterative Method is better than Adomian Decomposition Method.

\section{Acknowledgements}

The authors would like to thank the Rector of Universitas Padjadjaran for the project financial support through RDDU (Doctoral Dissertation Research of Universitas Padjadjaran) 2019 with contract number 5880/UN6.D/LT/2019. Also, the author would like to thank Universiti Malaysia Terengganu for this excellent collaboration work and support of this research publication.

\section{References}

Adomian, G. (1988). A review of the decomposition method in applied mathematics. Journal of Mathematical Analysis and Applications, 135(2), 501-544.

Anidiobu, G. A., Okolie, P. I. P., \& Oleka, D. C. (2018). Analysis of inflation and its effect on economic growth in Nigeria. Journal of Economics and Finance, 9(1), 28-36.

Gbadamosi, B., Adebimpe, O., Akinola, E. I., \& Olopade, I. A. (2012). Solving Riccati equation using Adomian decomposition method. International Journal of Pure and Applied Mathematics, 78(3), 409-417.

He, J. H. (1999). Variational iteration method-a kind of non-linear analytical technique: some examples. International Journal of Non-linear Mechanics, 34(4), 699-708.

Merdan, M. (2012). On the solutions fractional Riccati differential equation with modified Riemann-Liouville derivative. International Journal of Differential Equations, 17.

Podlubny, I. (1998). Fractional differential equations: an introduction to fractional derivatives, fractional differential equations, to methods of their solution and some of their applications. Elsevier.

Saad, K. M., Al-Shomrani, A. A., Mohamed, M. S., \& Yang, X. J. (2016). Solving fractional order logistic equation by approximate analytical methods. International Journal of Open Problems in Computer Science and Mathematics, 238(4025), 1-16.

Sukono, Subartin, B., Ambarwati, Saputra, J., Hidayat, Y. (2019). Forecasting model of gross regional domestic product (GRDP) using backpropagation of Levenberg-Marguardt method. Industrial Engineering and Management Systems, 18(3), 530-540.

Sukono,, Albra, W., Zulham, T., Saputra, J., Subartini, B., Thalia, F. (2019). The effect of gross domestic product and population growth on $\mathrm{CO} 2$ emissions in Indonesia: An application of the ant colony optimisation algorithm and cobbdouglas model. International Journal of Energy Economics and Policy, 9(4), 313-319.

Tarasov, V. E. (2011). Fractional dynamics: applications of fractional calculus to dynamics of particles, fields and media. Springer Science \& Business Media.

Tarasov, V. E., \& Tarasova, V. V. (2016). Long and short memory in economics: fractional-order difference and differentiation, IRA-International Journal of Management and Social Sciences, 5(2), 327-334.

Tarasov, V. E., \& Tarasova, V. V. (2016). Marginal utility for economic processes with memory. Almanac of Modern Science and Education, 7(109), 108-113.

Tarasov, V. E., \& Tarasova, V. V. (2021). Economic Dynamics with Memory: Fractional Calculus Approach (Vol. 8). Walter de Gruyter GmbH \& Co KG.

Tarasova, V. V., \& Tarasov, V. E. (2016). Criteria of hereditarity of economic process and the memory effect. Molodoj Uchenyj (Young Scientist), 14(118), 396-9.

Tarasova, V. V., \& Tarasov, V. E. (2016). Economic accelerator with memory: discrete time approach. arXiv preprint arXiv: 1612.07913.

Tarasova, V. V., \& Tarasov, V. E. (2016). Economic indicator that generalises average and marginal values. Ekonomika $i$ Predprinimatelstvo (Journal of Economy and Entrepreneurship), (11-1), 76-1.

Tarasova, V. V., \& Tarasov, V. E. (2016). Fractional dynamics of natural growth and memory effect in economics. European Research, 12(23), 30-37.

Valentina, T., \& Vasily, T. (2016). Economic accelerator with memory: discrete time approach. Проблемь современной науки и образования, 36(78).

Volterra, V. (2005). Theory of functionals and of integral and integro-differential equations. Dover Publications.

Wainwright, K. (2005). Fundamental methods of mathematical economics. McGraw-Hill/Irwin.

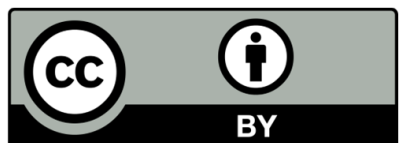

(C) 2022 by the authors; licensee Growing Science, Canada. This is an open access article distributed under the terms and conditions of the Creative Commons Attribution (CC-BY) license (http://creativecommons.org/licenses/by/4.0/). 